\title{
Microbiota Dynamics in Semi-Solid Fermentation of Light-Flavor Liquor with Corn as Main Material by Illumina Miseq Sequencing
}

Xiao Liu

China Agricultural University

Rufei Ma

China Agricultural University

Jinrong $\mathrm{Hu}$

China Agricultural University

Xia Zhou

China Agricultural University

Mengya Wang

China Agricultural University

Jingsheng Zhang

China Agricultural University

Ping Liu ( $\sim$ liuping@cau.edu.cn )

China Agricultural University

\section{Research article}

Keywords: Light-Flavor Liquor, Semi-solid Fermentation, Fermented Grains, Microbial Diversity, Highthroughput sequencing

Posted Date: October 29th, 2020

DOI: https://doi.org/10.21203/rs.3.rs-97516/v1

License: (c) (1) This work is licensed under a Creative Commons Attribution 4.0 International License. Read Full License 


\section{Abstract}

Background: Light-flavor liquor, with ethyl acetate as the main aroma component, is regarded as one of the four basic types in china. The traditional solid-state fermentation has a long period and a complicated process, but the content of esters and acids in liquid fermentation is not as good as that in solid-state fermentation. In this study, according to the methods of solid fermentation and liquid fermentation, the semi-solid fermentation of light-flavor liquor was simulated with optimized fermentation conditions, using corn and sorghum as brewing materials (corn: sorghum=7: 3). Furthermore, the bacterial and fungal community were investigated by high-throughput sequencing.

Results: The physical and chemical results showed that the content of reducing sugar, alcohol and ethyl acetate first increased and then decreased, while the contents of reducing sugar, alcohol and ethyl acetate reached the peak at $1 \mathrm{~d}, 11 \mathrm{~d}$ and $20 \mathrm{~d}$, respectively. The Illumina MiSeq Sequencing results showed that Lactobacillaceae and Leuconostocaceae were the dominant bacteria on the family level. Moreover, Pediococcus and Weissella were the main bacteria on the genera level. Meanwhile, 4 fungal phyla were identified with Ascomycota (83.6-99.99\%) as the dominant phyla. There were 5 species of fungi with the relative abundance over $1.00 \%$, with Saccharomyces cerevisiae and Saccharomycopsis fibuligera were the main species. From the 3rd day, the relative abundance of Saccharomycopsis fibuligera decreased rapidly to $2.4 \%$, while Saccharomyces cerevisiae (up to $99.2 \%$ ) became the absolute dominant fungus species. The results of Redundancy analysis (RDA) showed that starch and reducing sugar had a significant influence on Pediococcus. Saccharomycopsis fibuligera was most affected by starch. In addition, Saccharomyces cerevisiae was most affected by alcohol.

Conclusions: Saccharomycopsis fibuligera and Pediococcus were positively correlated to starch and reducing sugar, highlighting their important roles in the starch saccharification. Saccharomyces cerevisiae contributed to the glycolysis and esterification $\varangle$ because they were positively correlated to alcohol and total acid. Nevertheless, Saccharomyces cerevisiae, as the dominant fungus, had maintained a high abundance during the whole fermentation. This study could provide new insights to design new processes for semi-solid fermentation and improve the quality and controllability of liquor production.

\section{Background}

Chinese liquor is a traditional fermented food in China. With a history over 5,000 years, it has become one of the oldest liquor in the world. Chinese liquor enjoys a high reputation worldwide and plays an indispensable role in the history, culture and daily life of China [1]. In the long process of development, Chinese liquor has formed its unique brewing technology and style quality. Together with English whiskey, French brandy, Indian rum, Russian vodka and Dutch gin, they are known as " six distilled spirits of the world" $[2,3]$.

Chinese liquor is traditionally fermented by grains such as sorghum, corn, wheat, glutinous rice as fermenting materials, added with culture starters, and fermented by complex fermentation processes in 
the form of solid, semi-solid or liquid [4]. Chen et al. [5] studied the effects of different kinds of brewing materials of light-flavor liquor, and concluded that fermentation methods and raw materials had a great influence on the flavor of liquor. Compared with traditional liquid liquor of fermentation, the content of esters and acids in high-quality solid liquor is higher, which is an important factor of strong flavor of solid liquor [6]. However, solid fermentation has a long period and a complicated process. Semi-solid fermentation is more efficient than solid fermentation in mass and heat transfer, as well as easier to utilize the nutrients in fermented grains. Ma et al. [7] innovatively optimized the semi-solid fermentation process of light-flavor liquor, with the raw materials of corn and sorghum.

Good liquor comes from good starter. Daqu is an essential fermentation starter of traditional oriental fermented foods, such as soy sauce [8], vinegar [9] and rice wine [10]. Generally, Daqu is mainly divided into three different types in Chinese traditional liquor manufacturing: light flavor, strong flavor and soy sauce flavor [11]. Daqu plays a significant role in the brewing process of liquor [12]. At present, the microbial community structure of three types of Daqu has been reported [6, 13-15]. Fen Chiew Group, Luzhou laojiao group and Moutai Group are famous manufacturers of the representative light flavor liquor (Fen flavor), strong flavor liquor (Luzhou flavor) and soy sauce flavor liquor (Moutai flavor) respectively in China. The compound aroma with ethyl acetate mainly is the typical characteristic of lightflavor liquor [16]. Microbial community structure in fermented grains directly determined the quality and flavor of the liquor. Bacteria, yeasts, and molds play an irreplaceable role in the fermentation process [17, 18]. Symbiosis, competition, and inhibition among strains of microorganisms occurred in fermented grains, which resulted in the diversity and complexity of microorganisms [19]. At present, there are many kinds of researches on the microbial dynamics of traditional light-flavor liquor [20,21].

Besides, the traditional culture techniques have been used to analyze the community composition and quantity changes of microbes such as starters, fermented grains and pit mud in soy sauce flavor, strong flavor and light-flavor,, but there are still certain limitations [15, 22-25]. Liu et al. [26] used the polymerase chain reaction-denaturing gradient gel electrophoresis (PCR-DGGE) and single strand conformation polymorphism (SSCP) technology to study the main functional microorganisms of light-flavor liquor and isolated and identified of Saccharomycopsis fibuligera. Zhang et al. [11] studied the characterization of microbial community in Chinese liquor fermentation starters Daqu using nested PCR-DGGE. Lei [27] conducted a preliminary analysis on the microbial community structure of light-flavor liquor by Illumina Miseq sequencing and found that the dominant microorganisms were shared by Acetobacteraceae and Candida in Daqu and fermented grains, but most dominant microorganisms were different.

High-throughput sequencing technology has significantly increased the throughput and sensitivity compared to other sequencing technologies [28, 29]. Hu et al. [30] analyzed different qualities of pit mud by high-throughput sequencing method and found that the quality of pit mud was related to Lactobacillus, Clostridium, and Methanobacterium. Hong et al. [31] researched the microbiota composition of Chinese Rice Wine by both metagenomic sequencing and 16S rDNA sequencing, and found that the results obtained by the two methods were different. The results of $16 \mathrm{~S}$ rDNA sequencing demonstrated that the quality of yeast starter and liquor correlated with microbial taxonomic 
composition, which was exemplified by our finding that liquor spoilage resulted from a high proportion of genus Lactobacillus. However, the results of metagenomic sequencing revealed that liquor spoilage was due to rapid growth of Lactobacillus brevis at the early stage of fermentation, and metabolisms of microbes influence the liquor quality. Li et al. [21] studied the microorganisms of light-flavor liquor based on ribosome high-throughput sequencing analysis, concluded that the bacterial population in the fermentation process was mainly Lactobacillaceae, and the fungal population was mainly Saccharomycetaceae and Saccharomycopsidaceae. Also, more realistic microbial population information could be detected by high-throughput sequencing methods compared to denaturing gradient gel electrophoresis (DGGE) [24]. Furthermore, high-throughput sequencing technology has been widely applied in other aspects, such as the microbial diversity of strong flavor liquor, the bacterial succession of cheese products [32], and the diversity of fungi in soy sauce flavor liquor.

This study endeavored to combine the advantages of traditional solid fermentation and liquid fermentation, using corn and sorghum as brewing materials, to brew light-flavor liquor by a semi-solid method. In addition, combined with high-throughput sequencing to study the microorganisms of fermented grains in the fermentation process, thereby optimizing the semi-solid brewing process, improving the controllability and quality of light-flavor liquor.

\section{Methods}

\subsection{Sampling}

Semi-solid fermentation of light-flavor liquor was simulated with saccharification and fermentation, using corn and sorghum as brewing materials (corn: sorghum $=7: 3$ ) and Daqu (from Taiyuan Shanxi) and flavoring strain (from Angel Yeast Co., Ltd.) as saccharification starters. The corn flour and sorghum flour (corn: sorghum $=7: 3$, corn $=3000 \mathrm{~g}$, sorghum $=750 \mathrm{~g}$ ) were weighed, respectively, and mixed uniformly with $60 \%$ water (measured as dry material, $2250 \mathrm{~g}$ ). The mixture was moistened for $0.5-3 \mathrm{~h}$ at room temperature, steamed for about $1 \mathrm{~h}$ by water vapor, and cooled to room temperature, then well-mixed with $20 \%$ (750 g) Daqu, 3\% (112.5 g) flavoring strain and 300\% water of dry material. The mixture was evenly mixed and divided into 30 parts for further fermentation. The fermentation temperature was $27^{\circ} \mathrm{C}$ on the first day, $32{ }^{\circ} \mathrm{C}$ on the second day, $26^{\circ} \mathrm{C}$ on the $3-7$ days, and $25^{\circ} \mathrm{C}$ on the $8-20$ days. Moreover, during different fermentation stages of $0,1,3,5,7,9,11,14,17$ and 20 days, three paralleled fermented grains were selected for sampling each time, respectively. All the samples were stored at $-80{ }^{\circ} \mathrm{C}$ for the further analysis.

\subsection{Chemical and physical property analysis}

The Daqu, used in this experiment, was purchased from Taiyuan Shanxi, and was determined after analysis of six kinds of Daqu after pre-process optimization experiments. The physical and chemical properties of Daqu were determined, including water, starch, acidity, fermentation power, liquefaction power, saccharification power, esterification power, protease activity, etc. Besides, bacteria, bacilli, yeast, 
molds in Daqu were also counted [14]. Analysis of the microbial community in fermented grains on the 0 day by high-throughput sequencing could indicate the initial inoculation.

The fermented grains were ultrasonically treated at $0{ }^{\circ} \mathrm{C}$ for $30 \mathrm{~min}$, then centrifuged at $4{ }^{\circ} \mathrm{C}$ for $5 \mathrm{~min}$, the obtained supernatant was used to determine the content of reducing sugar, ethanol and ethyl acetate. Reducing sugar was analyzed by using the 3, 5-dinitrosalicylic acid (DNS) method. Alcohol was measured by alcohol meter according to Chinese national food safety standards. Starch was extracted from the fermented grains by acid hydrolysis $(20 \% \mathrm{HCl}, \mathrm{v} / \mathrm{v})$ for $30 \mathrm{~min}$. After the $\mathrm{pH}$ value of the hydrolysate was adjusted to 7.0 with $20 \%(\mathrm{w} / \mathrm{v}) \mathrm{NaOH}$, the total reducing sugar was determined. Starch content was estimated by calculating the difference between total reducing sugar and the original reducing sugar. All the samples were analyzed in triplicate.

\subsection{DNA extraction}

All samples were stored at $-80^{\circ} \mathrm{C}$ before deoxyribonucleic acid (DNA) extraction. Moreover, the three copies of the samples were well mixed as one sample. The genomic DNA was extracted from samples taken from the different fermentation stages using Soil DNA Kit (MoBio Laboratories Inc.) following the manufacturer's protocol. Total DNAs were precipitated with isopropyl alcohol, then they were washed with $95 \%$ ethanol and finally dissolved in water. The DNA quality and quantity were determined by agarose gel electrophoresis (1\% w/v agarose in $0.5 \times$ TAE buffer) (Bio-Rad, USA) [10, 33, 34].

\subsection{PCR amplification and Illumina MiSeq sequencing analysis}

For prokaryotes, universal primer 338F (5'-ACT CCT ACG GGA GGC AGC AG-3') and 806R (5'- GGA CTA CHV GGG TWT CTA AT-3') were used to amplify the V3-V4 hypervariable region of the 16S rRNA genes.

For eukaryotes, the ITS2 region of fungal rRNA gene was amplified by using universal primer ITS2 (5'- GCT GCG TTC TTC ATC GAT GC-3') and ITS1 (5'- GAA CCW GCG GAR GGA TCA- 3'). Primer 338F and ITS2 were added with barcodes.

Polymerase chain reaction (PCR) amplification and sequencing were described in detail previously [20]. The amplified PCR products were analyzed through a $2 \%(\mathrm{w} / \mathrm{v})$ agarose gel and purified by QIAquick Gel Extraction Kit (Qiagen, Germany). The concentrations of PCR purified products were quantified by NanoDrop 2000 spectrophotometer (Thermo, USA). Subsequently, the PCR products of all purified samples were constructed for a PCR amplicon library, and then sequenced by the Illumina MiSeq Platform of Majorbio I-Sanger.

\subsection{Sequencing data analysis}

Sequencing data analysis was performed using the free online platform of the Majorbio I-Sanger Cloud Platform (www.i-sanger.com). MiSeq sequencing obtained double-ended sequence data. The original data was optimized with software of FLASH and Trimmomatic based on overlap relationship between PE reads and barcode and primer sequences at both ends of the sequence. The optimized sequences (Tags) 
were clustered at $97 \%$ similarity level by the software of Usearch (vsesion $7.0 \mathrm{http}$ : //drive5.com/uparse/), and the operational taxonomic units (OTUs) were divided. Then the species classification was obtained according to the sequence composition of operational taxonomic unit (OTU) for subsequent analysis. Community composition analysis was plotted using the R Programming Language.

\section{Results}

\subsection{Chemical and physical properties during fermentation}

Ethyl acetate is the characteristic substance of light-flavor liquor. Fermented grains with different fermentation days were distilled, and the content of ethyl acetate in distilled liquor was detected (Fig. 1). With the extension of fermentation time, the content of ethyl acetate gradually increased, and reached the peak value after fermentation for 20 days. Subsequently, the content of ethyl acetate showed a decreasing trend.

The starch content in fermented grains decreased with the fermentation (Fig. 2), and the starch content decreased the fastest ( $0-7$ days) in the early stage of fermentation, and then decreased moderately in the middle and late stage (7-20 days). The change of reducing sugar concentration in fermented grains reflected the balance and coordination of saccharification and fermentation speed to some extent. The amount of residual sugar in fermented grains determined whether the fermentation of the fermented grains is natural. As shown in Fig. 2, with the fermentation, the content of reducing sugar in the fermented grains increased first and then decreased, but it remained relatively less and stable. Alcohol was the main product of fermented grains fermentation, which was mainly produced during the main fermentation period. The amount of production was also the main indicator for identifying the quality of fermented grains, which was also an important data to decide whether the fermentation is natural. As can be seen from Fig. 2, the alcohol content gradually increased with the progress of fermentation, which increased significantly in 0-5 days until it reached the maximum on the 11th day and then continued to decline. During the fermentation, ethanol was mainly produced by yeast metabolism, and secondly, lactic acid bacteria also produced a certain of ethanol when performing lactic acid fermentation [35].

\subsection{Microbial community structure and diversity}

After sequencing, the raw reads were filtered to remove potential erroneous sequences. A total of 509409 sequences were obtained after optimization of bacterial community, and the average length of optimized sequences was 449. Meanwhile, a total of 553063 sequences were obtained after optimization of fungal community, and the average length of optimized sequences was 435 for further study. OTU is the operational taxonomic unit (OTU). The sequences were clustered into an OTU based on $97 \%$ similarity. 140 OTUs and 105 OTUs were detected in bacterial and fungal community, respectively. In addition, there was a large difference in the OTU number between the pre- and post-fermentation period, indicating that the community composition of fermented grains was also changing with the content changes of starch, reducing sugar and alcohol during the fermentation. This trend was basically consistent with the change of physical and chemical properties. In the early stage of fermentation, a large amount of starch was 
consumed and alcohol was produced. The Coverage index of all samples was greater than 0.999 , indicating good community coverage.

According to the rarefaction curves, the Sobs curves did not reach a plateau, but the Shannon curves were approaching a parallel orientation to the horizontal axis (Fig. 3). The diversity of samples did not increase with the increase of sample sequencing depth. The results showed that the sequencing data could reflect the diversity of community microorganisms in different fermentation days, and demonstrated that the sequencing data was sufficient to evaluate the whole population.

\subsection{Microbial community compositions}

The bacterial community structure and succession during the semi-solid fermentation of the light-flavor liquor were measured by high-throughput sequencing technology. The results were shown in Fig. 4 and Fig. 5. The results of Fig. 4 showed that Firmicutes and Proteobacteria dominated in fermented grains. The results of Fig. 5 showed that there were 19 bacterial genera in the fermented grains with relative abundance over $1 \%$ in different fermentation stages. In addition, the main bacteria included Pediococcus, Weissella, Enterobacter, Cronobacter, and unclassified Lactobacillaceae with the relative abundance of 94.23-99.21\%. The remaining genera, namely Vogesella, Novosphingobium, Microbacterium, Curvibacter, Chryseobacterium, Acinetobacter and other bacterial genera accounted for about $4.80 \%$. The main bacterial genera of the fermented grains at different stages of the whole fermentation process were generally similar, but the relative abundance of each fungus showed fluctuating changes. Lactic acid bacteria were the dominant bacterias, mainly including Pediococcus, Weissella, Lactobacillus, etc. Among that, Pediococcus were the dominant genera of the fermentation system, and its relative abundance always maintained at a high level throughout the fermentation process.

Obviously, the genera Pediococcus had always occupied a relatively high abundance and showed fluctuating changes throughout the fermentation process. The relative abundance was $50.73 \%$ on the 0 day, and then increased to $74 \%$ on the 3 day. It also fell to $50.78 \%$ on the 7 day, rose to $63.45 \%$, and then decreased by $41.75 \%$. The relative abundance at the final fermentation was $54.40 \%$. In particular, the relative content of Acinetobacter increased sharply, standing at $32.90 \%$ and $12.12 \%$ on the 7 day and 17 day, respectively. The relative content of Novosphingobium was the highest on the 11 day $(5.77 \%)$.

Meanwhile, the relative abundance on family level was identified during different fermentation stages. The results suggested that 14 families were detected on family level, including Lactobacillaceae, Leuconostocaceae, Enterobacteriaceae, Sphingobacteriaceae, Microbacteriaceae, Methylobacteriaceae, Caulobacteraceae, Flavobacteriacere, Sphingomonadaceae, Moraxellaceae, Neisseriaceae. The dominant microorganisms in fermented grains were Lactobacillaceae and Leuconostocaceae throughout the whole fermentation process, but their relative abundance kept constantly changing. The relative abundance of the Leuconostocaceae fluctuated, reaching $7.98 \%$ on the 7 day and $34.32 \%$ on the 11 day. At the end of

the fermentation, the relative abundance of Neisseriaceae was the largest, and that of Moraxellaceae was the highest on the 7 day. 
The results showed that on the phylum level Ascomycota and Basidiomycota were the main fungi in fermented grains during the fermentation (Fig. 7). Among them, Ascomycota was the dominant fungal phyla, accounting for $83.62-99.98 \%$ of the total abundance. On the 0 day of fermentation, $83.62 \%$ of the fungi in fermented grains belonged to Ascomycota, and the rest belonged to the Basidiomycota. As the fermentation progressed, the relative abundance of Basidiomycota decreased, and fell to $1.24 \%$ on the 1 day of fermentation. From the 3 day to the end of fermentation, the relative abundance of Ascomycota was more than $99 \%$, which became the dominant fungal phyla in fermented grains.

The results of fungi in fermented grains during semi-solid fermentation showed that there were mainly five species of fungi with relative abundance of more than $1.00 \%$ on species level, namely Saccharomyces cerevisiae, Saccharomycopsis fibuligera, Cryptococcus diffluens, Rhodosporidium fluviale and Cryptococcus albidus. The fungal community structure was significantly different from the other stages in the initial stage of fermentation. On the 0 day of fermentation, five fungal species were identified, of which the dominant fungal species were Saccharomycopsis fibuligera, followed by Cryptococcus diffluens, and the relative abundance was $80.02 \%$ and $11.44 \%$, respectively. While the relative abundance of Saccharomyces cerevisiae was relatively small, accounting for only $0.29 \%$. With the fermentation, the relative abundance of Saccharomycopsis fibuligera decreased rapidly to $2.38 \%$ on the 3 day, while that of Saccharomyces cerevisiae rapidly increased to $96.94 \%$. Saccharomyces cerevisiae had become the dominant fungal species in fermented grains and remained an absolute dominant position until the end of fermentation.

\subsection{Effects of environmental factors on microbial community structure}

In this study, RDA analysis was used to study the relationship between environmental factors and microbial community. The results showed that the ethanol, total acid, starch and reducing sugar had obvious effects on bacterial community, and starch had the strongest effect, followed by reducing sugar (Fig. 9). The relative abundance of Weissella and Acinetobacter was positively correlated with alcohol and total acid. When the alcohol content was high or total acid was increased, the contents of Weissella and Acinetobacter would rise. Enterobacter and Pediococcus were positively correlated with starch and reducing sugar in environment, but negatively correlated with alcohol and total acid.

The results showed that the ethanol, total acid, starch and reducing sugar also had significant effects on fungal community (Fig. 10). However, the effect of environmental factors on fungal community was different from that of bacterial community. The starch had the strongest influence and alcohol was the second. Among them, reducing sugar had a great influence on bacterial community, but it had the least impact on fungal community, which may be caused by the utilization of reducing sugar by fungi and bacteria. The relative abundance of Saccharomycopsis fibuligera and Rhodotorula mucilaginosa was positively correlated with starch and reducing sugar. Among them, starch had a greater effect on Saccharomycopsis fibuligera, while starch and reducing sugar had less effect on Rhodotorula 
mucilaginosa. Saccharomyces cerevisiae was positively correlated with alcohol or total acid in the environment, where the effect of alcohol was slightly greater than the effect of total acid.

\section{Discussion}

\subsection{Chemical and physical properties}

In this study, combined with the methods of solid fermentation and liquid fermentation, semi-solid fermentation of light-flavor liquor was simulated with corn and sorghum as raw materials. The dynamics of microbial community in fermented grains during fermentation were studied by high-throughput sequencing.

The volatile compounds in fermented grains mainly include alcohols, aldehydes, acids and esters, etc., but ethyl acetate is the main the volatile components in the light flavor liquor. Among them, there are mainly two ways to form esters: the esterification of alcohol and acetic acid and the metabolism of ester yeasts. Due to acetic acid and ethanol are the precursor substances of esterification, a lot of ethanol and acetic acid are consumed during the esterification. As the fermentation progressed, the content of ethyl acetate in fermented grains increased continuously, reaching a maximum of 20 days, and then decreased continuously. It was speculated that in the early stage of fermentation, nutrients were abundant, and dominant microorganisms multiplied continuously, thereby continuously producing metabolites such as esters. In the late fermentation process, nutrients in the fermentation system were insufficient (Fig. 2), the microbial structure changes, while some of the microbials consume ethyl acetate in the system for survival $[1,30]$.

The fermentation process is a process in which starch macromolecules undergo three stages of saccharification, glycolysis, and esterification to produce aroma components such as alcohol and organic acids. Starch is the material basis for alcohol fermentation during fermentation. Starch is converted into other sugars, organic substances, etc. by saccharification of amylase and glucoamylase, thereby providing a material basis and energy source for the growth and metabolism of microorganisms, so that the fermentation process can be carried out normally. The change of starch content may be due to the rich nutrition in the early stage of fermentation, microorganisms in fermented grains made full use of the products of starch decomposition for metabolism, resulting in a rapid decline of starch. With the development of fermentation, the change of environmental factors, the increase of acids and esters, and the aggravation of anaerobic environment would affect the metabolism of microorganisms. Therefore, the rate of decrease of starch content in the late stage of fermentation decreased. During fermentation, saccharification proceeded simultaneously with fermentation, maintaining a state of relative dynamic equilibrium. Reducing sugar was obtained by amylase degradation of starch, which could provide energy to microorganisms while being used by yeast to produce alcohol. The trend of reducing sugar could be explained by the fact that the amylase hydrolyzed the starch to produce a large amount of reducing sugar due to the pre-fermentation period. Reducing sugar could not only provide energy for the growth of microorganisms, but also could be used by yeast for alcohol fermentation. In the early stage of 
fermentation, microorganisms were mainly in the stage of growth and reproduction, mainly using reducing sugar to provide energy, instead of yeast using reducing sugar for alcohol fermentation (Fig. 2 showed the low alcohol content in the early fermentation stage). At the time, the hydrolysis rate was greater than the utilization rate of reducing sugar, and therefore, the reducing sugar was in an accumulated rising state. After the yeast grew and reproduced rapidly, the alcohol fermentation rate was significantly accelerated, and the reducing sugar content was continuously decreased, then finally stabilized. During the early stage of fermentation, the starch was rapidly degraded, and the nutrition was extremely rich. Therefore, the yeast grew and multiplied, and the amount of ethanol began to increase. In the late stage of fermentation, it entered the esterification stage and consumed a part of alcohol for synthesizing aroma substances. In addition, in the middle and late stages of fermentation, when the amount of ethanol was accumulated to a certain extent, the diversification of the nutritional relationship would affect the growth and reproduction of the yeast to some degree, which would affect the convene of ethanol.

\section{2. microbial community}

Alpha values can be used to estimate the species diversity of microbial community species in the sample through several indices, including Observed Species, Chao1, ACE, Shannon and Simpson. The Chao1 index is used to measure the abundance index of the community. With the value increases, the abundance of the microbial community is correspondingly higher. The Simpson and Coverage indices reflect the diversity of the community [36]. The Alpha results indicated that the Coverage index of all samples was greater than 0.999 , showing a good community diversity. In the analysis of high-throughput sequencing data, Shannon plots and Sobs plots characterize the microbial diversity detected by samples at different sequencing depths. When the rarefaction curves flatten out, it indicates that the sequencing depth is sufficient and the microbial diversity in the sample can be detected substantially. When the slope of the rarefaction curves is high, it indicates that the sequencing depth is not enough [37, 38]. If the sequencing depth continues to increase, more microbial diversity can still be detected. Moreover, the Shannon plots and Sobs plots also proved that the sequencing depth was enough to cover the entire microbial community.

RDA analysis is mainly used to reflect the relationship between microbial community and environmental factors. Microbial community in the environment was affected by physical and chemical factors, which was an important reason for the variation of microbial abundance in fermented grains. The physical and chemical properties of fermented grains would change significantly during the fermentation process. Therefore, studying the interaction between microbial community structure and environmental factors could help to change the fermentation conditions to control the growth of beneficial microorganisms and inhibit the proliferation of harmful microorganisms. Wang studied the changes in physical and chemical properties of fermented grains during the fermentation process, but there is little research on the relationship between environmental factors and microbial community changes in the existing literature $[16,39]$. 
Wang [40] conducted high-throughput sequencing of microorganisms in fermented grains of light-flavor liquor, and the research results showed that a total of 22 phyla were detected in bacterial community, mainly including Firmicutes, Proteobacteria, Actinobacteria and Bacteroidetes. The results were completely consistent with this study (Fig. 4). Among them, Firmicutes and Proteobacteria took the dominated places in fermented grains. However, Wang [41] believed that the relative abundance of Firmicutes (91.73-99.73\%) in the late stage of fermentation was due to the decrease of bacterial population diversity and the succession of a single Firmicutes fermentation model. Li et al. [20] also thought that Firmicutes and Proteobacteria were the main bacterial populations after analyzing fermented grains in the fermentation process of light-flavor liquor through pyrosequencing. In addition, a large number of studies showed that Firmicutes and Proteobacteria were also the main bacterial community in the fermentation of strong flavor liquor [42], Moutai flavor liquor [33] and sesame flavor liquor [43], indicating that these bacterial community were the key microorganisms in the fermentation of Chinese liquor.

In this study, high-throughput sequencing results of bacteria showed that there were 19 genera of bacteria with the relative abundance of more than $1.00 \%$, among which Pediococcus, Weissella and Lactobacillus (a total of $91 \%$ ) were the dominant genera (Fig. 5). Pediococcus was the dominant bacteria in the fermentation system, which kept the relative abundance at a high level during the whole fermentation process. This was significantly different from the composition and structure of Lactobacillus in the solid fermentation of light-flavor liquor. Wu et al. [44] analyzed the evolution of Lactobacillus in the solid fermentation of light-flavor liquor, and the DGGE analysis indicated that Lactobacillus was the dominant genus in fermented grains. The results of Wang [41] also confirmed that both the old and new factories were dominated by Lactobacillus (68.89-97.72\%) in the middle and late stages of fermentation. Nevertheless, the results were not consistent with the results of this study. Correspondingly, the results in this study mainly included Pediococcus and Weissella. Among them, the Weissella could metabolize organic acids such as lactic acid and acetic acid to provide a synthetic precursor for flavor substances in Chinese liquor. The separation results of Ma [4] showed that the fermented grains were mainly composed of Pediococcus acidilactici, which showed $10 \%$ alcohol tolerance. Whereas, RDA results indicated that Pediococcus acidilactici might not contribute significantly to aroma components during the fermentation. Furthermore, it is also considered that most dominant lactobacillus in the fermentation process were lactobacillus of hetero fermentation, which could produce metabolic production of lactic acid, acetic acid, ethanol, etc. $[29,45,46]$.

Moreover, Li [47] analyzed microorganisms in the fermentation process of Fen liquor based on highthroughput sequencing of ribosome and considered that the major bacteria were mainly Lactobacillaceae. Lei [27] showed that Leuconostocaceae, Lactobacillaceae and Acetobacteraceae were absolute dominant bacteria. Among them, Leuconostocaceae, Lactobacillaceae were also dominant bacteria in this study. This result was confirmed in this experiment (Fig. 6).

Ascomycota generally predominates in many fermented foods. Wang et al. [40] showed that there were 8 fungal types on phylum level in the fermented grains of light flavor liquor by high-throughput sequencing, 
among which Ascomycota was the dominant fungal phyla in fermented grains (85.94-99.04\%). According to previous studies, Ascomycota was not only the dominant fungal community in fermented grains of light flavor liquor, but also the major fungus in a variety of fermented foods such as yellow wine [48], strong flavor liquor [43], Moutai-flavor liquor [22], vinegar [49], bread [50], etc. In addition, Li et al. believed that the structure of eukaryotic community in fermented grains was relatively stable on phylum level during the whole fermentation period. Since fermentation 3d, Ascomycota became the only dominant eukaryotic fungus in fermented grains [51], which was consistent with the results of this study (Fig. 6).

Li et al. [47] studied the microorganisms in the fermentation process of light-flavor liquor based on ribosome high-throughput sequencing analysis, concluded that the fungal population was mainly Saccharomycetaceae and Saccharomycopsidaceae, and the results were consistent with this study.

Saccharomycopsis fibuligera can hydrolyze starch [52], because of the property of secreting amylase, acid protease, and $\beta$-glucosidase, which is considered as the main amylolytic yeast during the fermentations of indigenous food involving cereals (like rice or sorghum) [53,54]. Therefore, Saccharomycopsis fibuligera usually dominates as the main amylolytic microorganisms and plays a crucial role at the beginning of the alcoholic fermentation, mainly helping to reduce the starch or polysaccharides into fermentable, low molecular weight sugars, such as maltotriose, dextrin, and maltose, and these could be subsequently hydrolyzed to glucose, which is the precursor of ethanol and other flavor compounds [55]. Wang et al. [56] studied the fermentation capacity and flavor components of the species isolated from a Moutai-flavor Daqu. Furthermore, the results also demonstrated that Saccharomycopsis fibuligera was beneficial to produce high contents of ethyl acetate, ethyl palmitate and other aroma components.

It had been reported that Saccharomycopsis fibuligera was widely present in various starters. At present, the research on the Saccharomycopsis fibuligera in the domestic brewing industry is mainly concentrated in the fields of brewing liquor such as yellow rice wine, rice wine and light flavor liquor. In these studies, the Saccharomycopsis fibuligera was usually expressed in the form of dominant fungi in starters [57]. Among them, the Saccharomycopsis fibuligera occupied the dominant position of the fungus in Fen Daqu, while Saccharomycopsis cerevisiae is less [58]. Saccharomycopsis fibuligera could secrete aamylase, glucoamylase, etc., which was the main starch-degrading bacteria in the fermentation process of starch as the main raw material [59].Therefore, we can speculate that the importance of Saccharomycopsis fibuligera in the brewing process of Chinese liquor is not the conversion of sugars into alcohol, but as the saccharification yeast involved in the hydrolysis of macromolecules such as starch in sorghum and other brewing materials. Thus, enough glucose is accumulated for growth and metabolism by other microorganisms, providing sufficient precursor material for ethanol production [20].

Studies had confirmed that during the period of Fen liquor brewing, the Saccharomycopsis fibuligera was more abundant in the early stage, and then gradually decreased. The result was also proved by this study (Fig. 8). The results could also be confirmed by RDA analysis (Fig. 10). In the late stage of fermentation, 
the alcohol content increased continuously, but the starch content continued to decrease. As a result, the Saccharomycopsis fibuligera was continuously decreased, but Saccharomyces cerevisiae became the dominant fungus in fermented grains and maintained the dominant position until the end of fermentation. This was similar to the change of yeast community in the brewing process of Fen liquor. In addition, studies had shown that Saccharomycopsis fibuligera had the property of producing highly active $\beta$-glucosidase, which could be applied to the liquor-making industry to improve the liquor quality [60].

Saccharomyces cerevisiae generally predominates during the alcoholic fermentations as it has a good capacity to grow in strict anaerobic conditions, whereas it is not an abundant yeast species in Daqu [61, 62]. Zheng et al. [6] studied the microbial diversity in Fen-Daqu by through PCR-DGGE analysis, but Saccharomyces cerevisiae was not detected. However, only one isolate of Saccharomyces cerevisiae was observed by culture-dependent approach. RDA analysis results showed a negative correlation between Saccharomyces cerevisiae and starch content and a little negative correlation with the content of reducing sugar. Due to the degradation of a large amount of starch in the early fermentation, the degradation products of reducing sugars such as glucose and fructose were produced. Chen [63] thought that excessive reducing sugar content would inhibit the proliferation of yeast. However, in the mixed fermentation process of molds and yeasts, the starch degraded to produce reducing sugar, and at the same time reducing sugar provided energy for the growth of the microorganisms and was continuously consumed, which would reduce the activity inhibition of reducing sugar on Saccharomyces cerevisiae and promote continuous fermentation. Furthermore, the research by Ma et al. [4] had demonstrated that Saccharomyces cerevisiae fermented despite low initial concentration, particularly because of the presence of competitive growth with fermentable sugars and higher alcohol tolerance.

In general, Saccharomyces cerevisiae is considered as the most critical microorganisms in the fermentation process because of its strong ability to competitively utilize fermentable glycogen and its alcohol tolerance and plays an important role in improving fermentation efficiency and promoting alcohol production. It also has the ability to replace the dominant position in the fermentation process [64]. In addition, compared with solid-state fermentation, the fungal diversity in the semi-solid fermentation process was less, especially some aroma-producing yeasts such as Pichia pastoris were not found in the fermentation process. Saccharomyces cerevisiae had a dominant position during the fermentation process. In addition, Ma et al. [4] also suggested that Saccharomyces cerevisiae was associated with some high alcohols and acids.

\section{Conclusions}

Based on the traditional solid-state fermentation of light-flavor liquor, this study simulated innovatively the semi-solid fermentation of light-flavor liquor with corn and sorghum as raw materials and optimized technological conditions. Combined with high-throughput sequencing technology, the bacterial and fungal community structure and diversity of fermented grains in semi-solid fermentation process of lightflavor liquor were detected, and the relationship between environmental factors and microbial dynamics 
was analyzed through RDA analysis. Similar to many studies, we found that during the whole fermentation process, the bacterial community was dominated by Firmicutes and Proteobacteria, while the fungal community was dominated by Ascomycota. On genera level, Pediococcus and Weissella were the main bacteria genera in the fermentation process. Besides, in terms of fungi, Saccharomyces cerevisiae and Saccharomycopsis fibuligera were the main fungal species on species level. In addition, microbial community structure and environmental factors interacted during fermentation.

In this study, some aromatic and ester producing yeasts such as pichia pastoris were not found. Furthermore, this paper provided new ideas for semi-solid fermentation that could shorten the fermentation period. In the future, pure strains such as Pichia pastoris could be added for strong fermentation or mixed fermentation could be conducted with superior strains to improve the quality of Chinese liquor.

\section{Abbreviations}

DGGE: Denaturing gradient gel electrophoresis; DNS: 3, 5-dinitrosalicylic acid; OTU: Operational taxonomic unit; OTUs: Operational taxonomic units; PCR: Polymerase chain reaction; PCR-DGGE: Polymerase chain reaction-denaturing gradient gel electrophoresis; RDA: Redundancy analysis; SSCP: Single strand conformation polymorphism

\section{Declarations}

\section{Ethics approval and consent to participate}

Not applicable

\section{Consent to publish}

The authors agree to submit this manuscript to BMC Microbiology.

\section{Availability of data and materials}

The original sequencing data supporting the conclusions of this article is available in the free online platform of the Majorbio I-Sanger Cloud Platform.

\section{Competing interests}

The authors declare that they have no competing interests. 


\section{Funding}

Not applicable

\section{Authors' Contributions}

$\mathrm{XL}$ performed the experiments, and wrote the manuscript. RM conceived and designed the study. MW and $\mathrm{XZ}$ revised the manuscript. JZ and JH participated in the planning and coordination of the study. PL conceived, designed and revised the manuscript. All authors read and approved the final manuscript.

\section{Acknowledgements}

We thank Jilin Alcohol Research Institute Co., Ltd for materials assistance and advice.

\section{References}

1. Du H, Fan W, Xu Y: Characterization of geosmin as source of earthy odor in different aroma type Chinese liquors. J Agric Food Chem 2011, 59(15):8331-8337.

2. Gao S Q: The impact of China's entry into WTO on China's liquor industry and countermeasures. Chinese Wine 2000, 14-16(In Chinese).

3. R DJC, Sánchez MJI, Roland V, Pat S, De Kimpe Norbert: Analysis of volatiles of malt whisky by solidphase microextraction and stir bar sorptive extraction. J Chromatogr A 2003, 985(1-2):221-232.

4. Ma RF, Sui L, Zhang JS, Hu JR, Liu P: Polyphasic Characterization of Yeasts and Lactic Acid Bacteria Metabolic Contribution in Semi-Solid Fermentation of Chinese Baijiu (Traditional Fermented Alcoholic Drink): Towards the Design of a Tailored Starter Culture. Microorganisms 2019, 7(5):147.

5. Chen B, He H K, Li A J, Wang D L, Liu G Y, Han X L: Effect of different grain varieties on the flavor of Fen-flavour Daqu Baijiu (Chinese liquor). Chinese Brewing 2017, 36: 22-26 (In Chinese).

6. Zheng XW, Yan Z, Han BZ, Zwietering MH, Samson RA, Boekhout T, Robert Nout MJ: Complex microbiota of a Chinese "Fen" liquor fermentation starter (Fen-Daqu), revealed by culture-dependent and culture-independent methods. Food Microbiol 2012, 31(2):293-300.

7. Ma R F, Ma Y B, Tong S S, Liu P: Optimization of corn brewing process of light-flavor liquor by liquid fermentation. Liq-Mak Sci Technol 2018, 293: 80-84 (In Chinese).

8. Devanthi PVP, Gkatzionis K: Soy sauce fermentation: Microorganisms, aroma formation, and process modification. Food Res Int 2019, 120:364-374.

9. Li S, Li P, Liu X, Luo L, Lin W: Bacterial dynamics and metabolite changes in solid-state acetic acid fermentation of Shanxi aged vinegar. Appl Microbiol Biot 2016, 100(10):4395-4411.

10. Liu SP, Mao J, Liu YY, Meng XY, Ji ZW, Zhou ZL, Ai-lati A: Bacterial succession and the dynamics of volatile compounds during the fermentation of Chinese rice wine from Shaoxing region. World $\mathrm{J}$ 
Microb Biot 2015, 31(12):1907-1921.

11. Zhang LQ, Wu CD, Ding XF, Zheng J, Zhou RQ: Characterisation of microbial communities in Chinese liquor fermentation starters Daqu using nested PCR-DGGE. World J Microb Biot 2014, 30(12):30553063.

12. Zheng Y, Zheng XW, Han BZ, Han JS, Nout M J R, Chen JY: Monitoring the ecology of Bacillus during Daqu incubation, a fermentation starter, using culture-dependent and culture-independent methods. J Microbiol Biotechn 2013, 23(5):614-622.

13. Luo H B, Li H, Huang Z G, Zhang Q, We, C H, Shen C P: Optimal conditions of PCR-SSCP technology for the analysis of microbial community in Luzhou-flavor Daqu. J Sichuan university Sci engineering 2009, 22: 72-74(In Chinese).

14. Ma R F, Ma Y B, Tong S S, Liu P: Analysis of physiochemical and biochemical indexes and volatile compounds of different fen-flavor Daqu. Liq-Mak Sci Technol 2018, 3: 70-75 (In Chinese).

15. Wang CL, Shi DJ, Gong GL: Microorganisms in Daqu: a starter culture of Chinese Maotai-flavor liquor. World J Microb Biot 2008, 24(10):2183-2190.

16. Wang H Y: Master's thesis. Jiangnan University, Wuxi, China, 2014.

17. Ward DM, Weller R, Bateson MM: 16S rRNA sequences reveal numerous uncultured microorganisms in a natural community. Nature 1990, 345(6270):63-65.

18. Xiang WL, Li K, Liu S, Xing YG, Li MY, Che ZM: Microbial succession in the traditional Chinese Luzhou-flavor liquor fermentation process as evaluated by SSU rRNA profiles. World J Microb Biot 2013, 29(3):559-567.

19. Li P, Lin WF, Liu X, Wang XW, Gan X, Luo LX, Lin WT: Effect of bioaugmented inoculation on microbiota dynamics during solid-state fermentation of Daqu starter using autochthonous of Bacillus , Pediococcus , Wickerhamomyces and Saccharomycopsis. Food Microbiol 2017, 61:82-93.

20. Li XR, Ma EB, Yan LZ, Meng H, Du XW, Zhang SW, Quan ZX: Bacterial and fungal diversity in the traditional Chinese liquor fermentation process. Int J Food Microbiol 2011, 146(1):31-37.

21. Li XR, Ma EB, Yan LZ, Meng H, Du XW, Quan ZX: Bacterial and fungal diversity in the starter production process of Fen liquor, a traditional Chinese liquor. J Microbiol 2013, 51(4):430-438.

22. Wu Q, Chen LQ, Xu Y: Yeast community associated with the solid state fermentation of traditional Chinese Maotai-flavor liquor. Int J Food Microbiol 2013, 166(2):323-330.

23. Chen MB, Yang DD, Qian ZW, Zhen D, Peng DH, Fang SL: Analysis of microorganisms and physicochemical properties in Zaopei during the fermentation of Chinese zhijiang-flavor liquor. African J. Biot. 2010, 9(25):3822-3874.

24. Zhang X, Zhao J, Du X: Barcoded pyrosequencing analysis of the bacterial community of Daqu for light-flavour Chinese liquor. Lett Appl Microbiol 2014, 58(6):549-555.

25. Liu MK, Tang YM, Guo XJ, Zhao K, Tian XH, Liu Y, Yao WC, Deng B, Ren DQ, Zhang XP: Deep sequencing reveals high bacterial diversity and phylogenetic novelty in pit mud from Luzhou Laojiao cellars for Chinese strong-flavor Baijiu. Food Res Int 2017, 102:68-76. 
26. Liu Z L, Cao J, Wang D L, Wang X L, Hu J H: Isolation and identification of Saccharomycopsis fibuligera from Fen-flavor Daqu and fermented grains. Chinese Brewing 2011, 38-41(In Chinese).

27. Lei ZH: Primary analysis on the fermented microorganism of Fen-flavor liquor by high-throughput sequencing. Food Ferment. Ind. 2015, 41(9):164-167.

28. Wang X C, Yang Z R, Wang M, Li W, Li S C: High-throughput sequencing technology and its application. China Biotechnol 2012, 32: 109-114 (In Chinese).

29. Song Z W, Du H, Zhang Y, Xu Y: Unraveling core functional microbiota in traditional solid-state fermentation by high-throughput amplicons and metatranscriptomics sequencing. Front Microbiol 2017, 8: 1294.

30. Hong XT, Chen J, Liu L, Wu H, Tan HQ, Xie GF, Xu Q, Zou HJ, Yu WJ, Wang L et al: Metagenomic sequencing reveals the relationship between microbiota composition and quality of Chinese Rice Wine. Sci Rep-UK 2016, 6(1).

31. Hu XL, Du H, Ren C, Xu Y: Illuminating Anaerobic Microbial Community and Cooccurrence Patterns across a Quality Gradient in Chinese Liquor Fermentation Pit Muds. APPL ENVIRON MICROB 2016, 82(8):2506-2515.

32. Carraro L, Maifreni M, Bartolomeoli I, Martino Maria E, Novelli E, Frigo F, Marino M, Cardazzo B: Comparison of culture-dependent and independent methods for bacterial community monitoring during Montasio cheese manufacturing. Res Microbiol 2011, 162(3):101-114.

33. Zhang Y, Zhu X, Li X, Tao Y, Jia J, He X: The process-related dynamics of microbial community during a simulated fermentation of Chinese strong-flavored liquor. Bmc Microbiol 2017, 17(1):196.

34. Wang L, Wang YY, Wang DQ, Xu J, Yang F, Liu G, Zhang DY, Feng Q, Xiao L, Xue WB et al: Dynamic changes in the bacterial community in Moutai liquor fermentation process characterized by deep sequencing. J I Brewing 2015, 121(4):603-608.

35. Xie Y Q, Zhong Y, Xie X, Wang W D: Roles \& functions of lactic acid bacteria in the production of liquor by solid fermentation. Liq-Mak Sci Technol 2008, 83-86.

36. Keylock CJ: Simpson diversity and the Shannon-Wiener index as special cases of a generalized entropy. Oikos 2005, 109(1).

37. Jung JY, Lee SH, Lee HJ, Seo H, Park W, Jeon CO: Effects of Leuconostoc mesenteroides starter cultures on microbial communities and metabolites during kimchi fermentation. Int J Food Microbiol 2012, 153(3).

38. Nam YD, Lee SY, Lim SI: Microbial community analysis of Korean soybean pastes by next-generation sequencing. Int J Food Microbiol 2012, 155(1-2):36-42.

39. Wu XH, Zheng XW, Han BZ, Vervoort J, Nout MJR: Characterization of Chinese Liquor Starter, "Daqu", by Flavor Type with H-1 NMR-Based Nontargeted Analysis. J Agr Food Chem 2009, 57(23):1135411359.

40. Wang X S: PhD thesis. Jiangnan University, Wuxi, China, 2018. 
41. Wang XS, Du H, Xu Y: Source tracking of prokaryotic communities in fermented grain of Chinese strong-flavor liquor. Int J Food Microbiol 2017, 244:27-35.

42. Zhang WX, Qiao ZW, Shigematsu T, Tang YQ, Hu C, Morimura S, Kida K: Analysis of the Bacterial Community in Zaopei During Production of Chinese Luzhou-flavor Liquor. J I Brewing 2015, 111(2):215-222.

43. Wang HY, Zhang XJ, Zhao LP, Xu Y: Analysis and comparison of the bacterial community in fermented grains during the fermentation for two different styles of Chinese liquor. $J$ Ind Microbiol Biot 2008, 35(6):603-609.

44. Wu L L, Wang H Y, Xu Y, Wang D: Differences of lactic acid bacteria community between soy sauce aroma style and light aroma style liquor fermentation. Microbiol 2013, 40: 2182-2188(In Chinese).

45. Song HM, Moon JS, Chang HC: Rapid manufacture and quality evaluation of long-term fermented kimchi ( mukeunji ) using Lactobacillus sakei SC1. Food Sci Biotechnol 2015, 24(5):1797-1804.

46. Salmeron I, Fuciños P, Charalampopoulos D, Pandiella SS: Volatile compounds produced by the probiotic strain Lactobacillus plantarum NCIMB 8826 in cereal-based substrates. Food Chem 2009, 117(2):265-271.

47. Li X R: PhD thesis. Fudan University, Shanghai, China, 2011.

48. Lv X C, Weng X, Huang R L, Zhang W, Rao P F, Ni L: Research on biodiversity of yeasts associated with Hongqu glutinous rice wine starters and the traditional brewing process. J Chinese Ins Food Sci Technol 2012, 12: 182-190(In Chinese).

49. Wang ZM, Lu ZM, Shi JS, Xu ZH: Exploring flavour-producing core microbiota in multispecies solidstate fermentation of traditional Chinese vinegar. Sci Reports 2016, 6(1).

50. Emilie L, Charlotte U, Judith L, Xavier D, Bernard O, Delphine S: Sourdough microbial community dynamics: An analysis during French organic bread-making processes. Food Microbiol 2016, 53(Pt A):41-50.

51. Li K M, Fu G M, Wu C F, Liu C M, Wan Y, Pan F, Deng F P: Dynamics of eukaryotic microbial community succession during the traditional fermentation of special-flavor liquor. Food Sci 2017, 38 : 131-136(In Chinese).

52. Wickerham LJ, Lockwood LB, Pettijohn OG, Ward GE: Starch Hydrolysis and Fermentation by the Yeast Endomycopsis Fibuliger. J Bacteriol 1944, 48(4):413-427.

53. Nie ZQ, Yu Z, Min W, Yue H, Wang YN, Luo JM, Niu DD: Exploring microbial succession and diversity during solid-state fermentation of Tianjin duliu mature vinegar. Bioresource Technol 2013, 148(8):325-333.

54. Saelim K, Dissara Y, H-Kittikun A: Saccharification of cassava starch by Saccharomycopsis fibuligera YCY1 isolated from Loog-Pang (rice cake starter). Songklanakarin J Sci Technol. 2008, 30.

55. Ma R F: Master's thesis. China Agricultural University, Beijing, China, 2019.

56. Wang X D, Chen M Z, Ban S D, Qiu S Y: Separation, Identification and functional characterization of yeast strains from Moutai-flavor Daqu, a traditional Chinese liquor fermentation starter. Food Sci 
2017, 38: 51-57(In Chinese).

57. Liu C, Xie G F, Sun J Q, Zou H J, Wang R Q, Qian B, Zang W, Zhou J D, Shou H R, Bai F Y: Research progress on the microorganisms of Chinese rice wine. Sci Technol Food Ind 2016, 37: 374-380.

58. Qiao X M, Zhao J L, Du X W, Zhang X H: Analysis of fungal community of qingxiang Daqu by barcoded pyrosequencing. Liq-Mak Sci Technol 2015, 28-31 (In Chinese).

59. Ismaya WT, Hasan K, Kardi I, Zainuri A, Rahmawaty RI, Permanahadi S, El Viera BV, Harinanto G, Gaffar S, Natalia D et al: Chemical Modification of Saccharomycopsis fibuligera R64 a-Amylase to Improve its Stability Against Thermal, Chelator, and Proteolytic Inactivation. Appl Biochem Biotech 2013, 170(1):44-57.

60. Sadoudi M, Tourdot-Maréchal R, Rousseaux S, Steyer D, Gallardo-Chacón JJ, Ballester J, Vichi S, Guérin-Schneider R, Caixach J, Alexandre H: Yeast-yeast interactions revealed by aromatic profile analysis of Sauvignon Blanc wine fermented by single or co-culture of non-Saccharomyces and Saccharomyces yeasts. Food Microbiol 2012, 32(2):243-253.

61. Nout MJR: Rich nutrition from the poorest-Cereal fermentations in Africa and Asia. Food Microbiol 2009, 26(7).

62. Urso R, Rantsiou K, Dolci P, Rolle L, Comi G, Cocolin L: Yeast biodiversity and dynamics during sweet wine production as determined by molecular methods. Fems Yeast Res 2008, 8(7).

63. Chen B: Master's thesis. Jiangnan University, Wuxi, China, 2014.

64. Sun Y T: Master's thesis. China Agricultural University, Beijing, China, 2014.

\section{Figures}

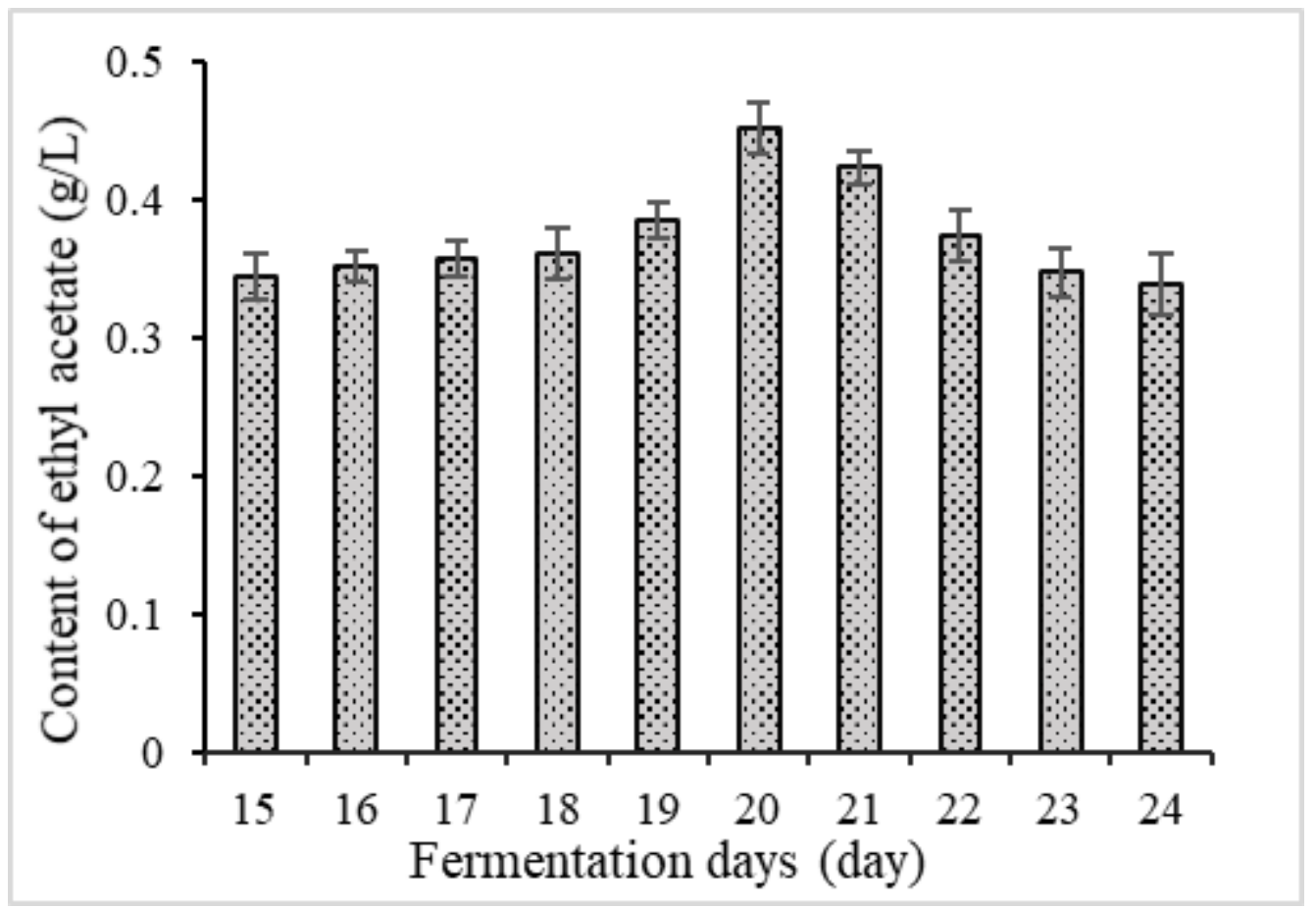

Figure 1 
Concentration of ethyl acetate in distilled base liquor during fermentation

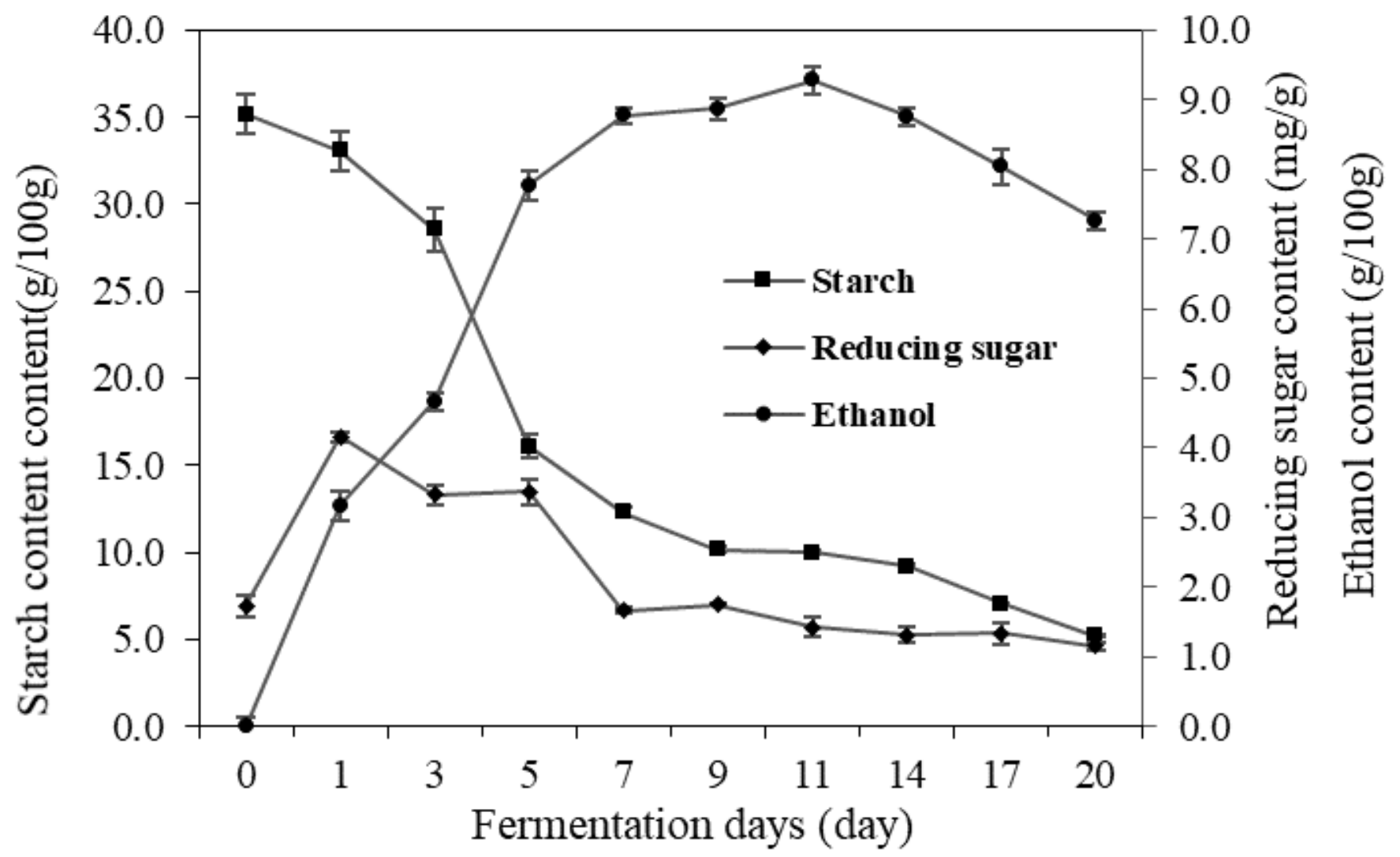

Figure 2

The content of starch, reducing sugar and ethanol of the fermented grains during fermentation process. 


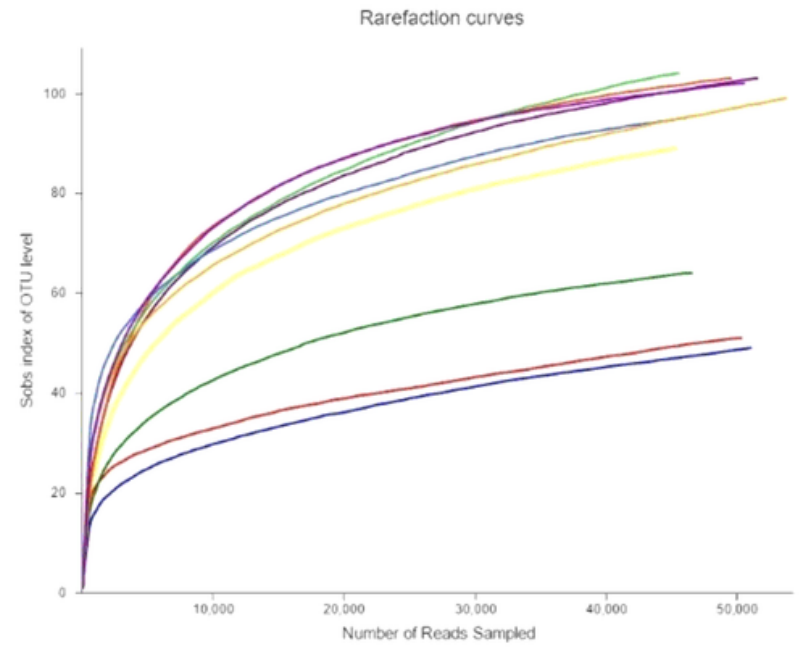

(a)

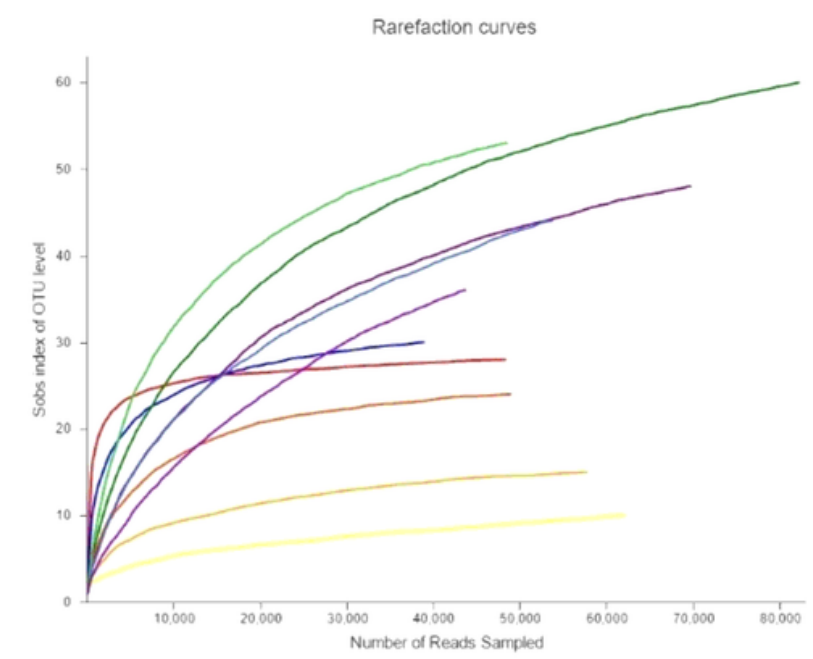

(c)

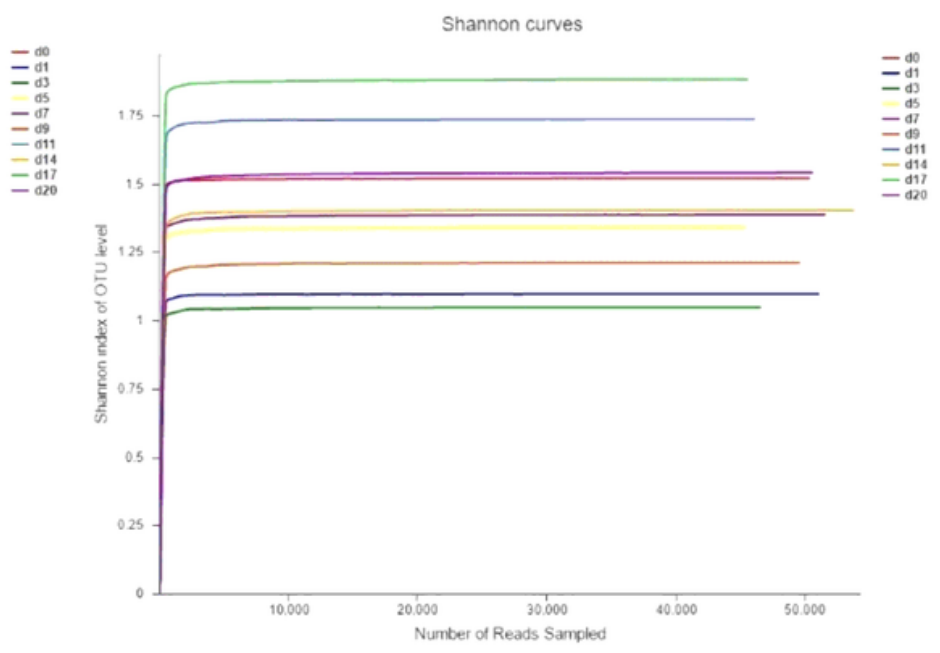

(b)

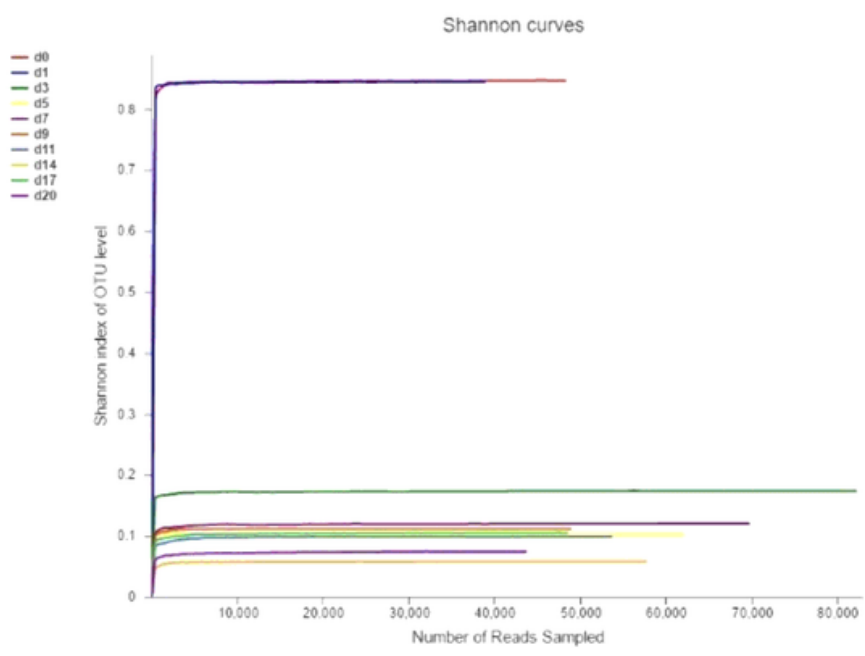

(d)

\section{Figure 3}

Rarefaction curves for samples of different days, based on the Sobs index and Shannon index. (a) Sobs index of bacterial, (b) Shannon index of bacterial, (c) Sobs index of fungal, (d) Shannon index of fungal. 
Community barplot analysis

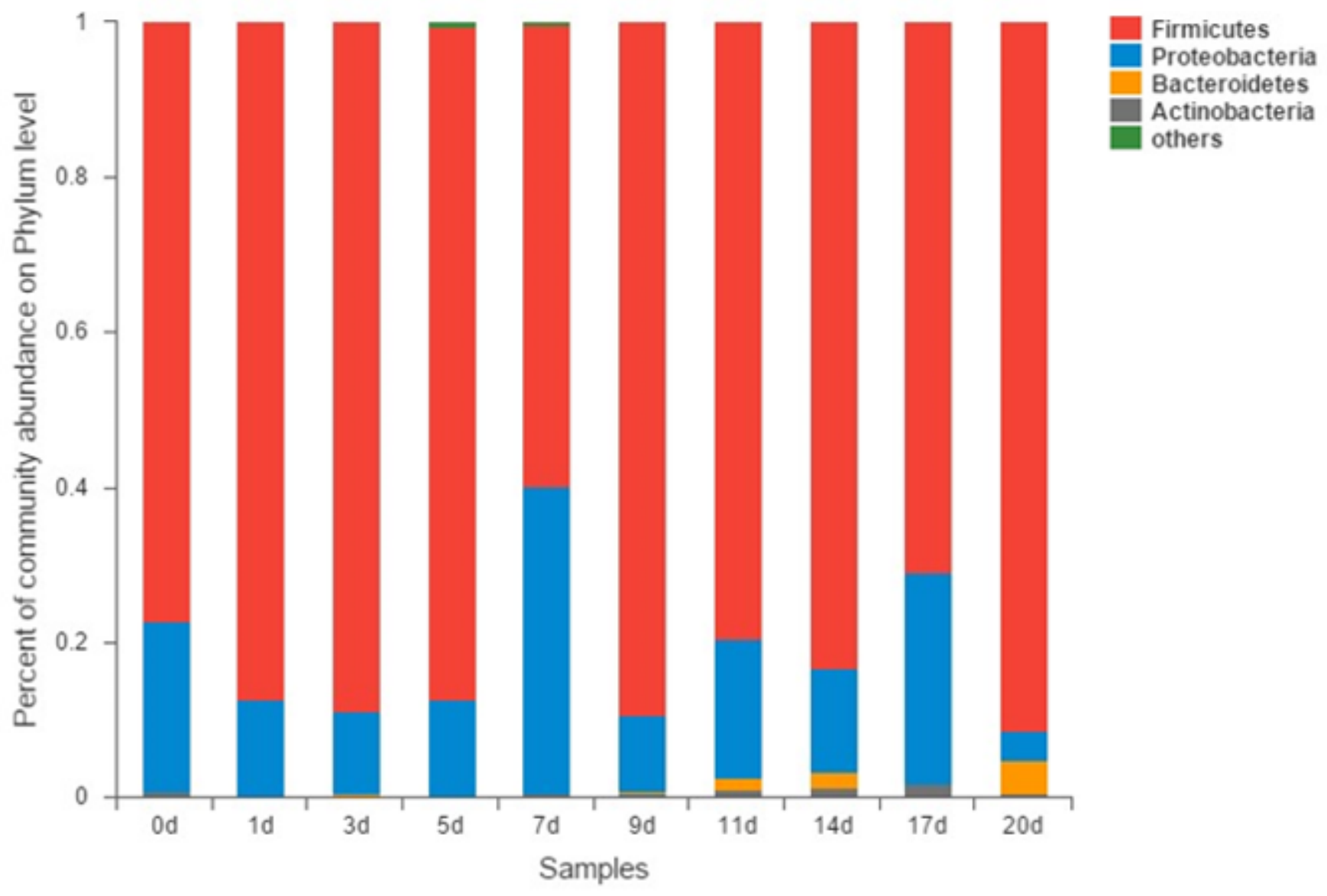

Figure 4

Relative abundance plots of bacterial community composition during the fermentation on Phylum level. 
Community barplot analysis

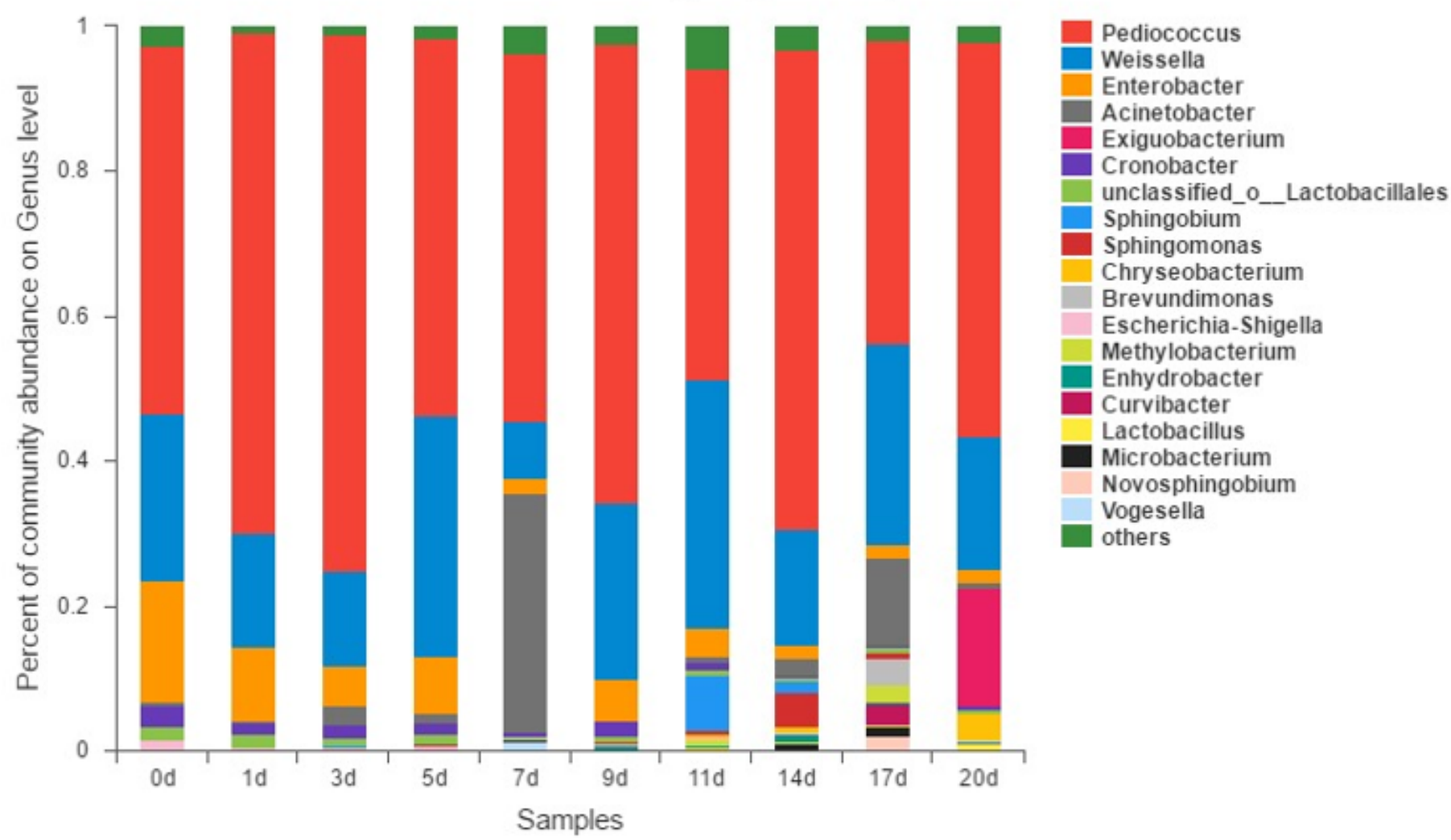

Figure 5

Relative abundance plots of bacterial community composition during the fermentation on genus level. 


\section{Community barplot analysis}

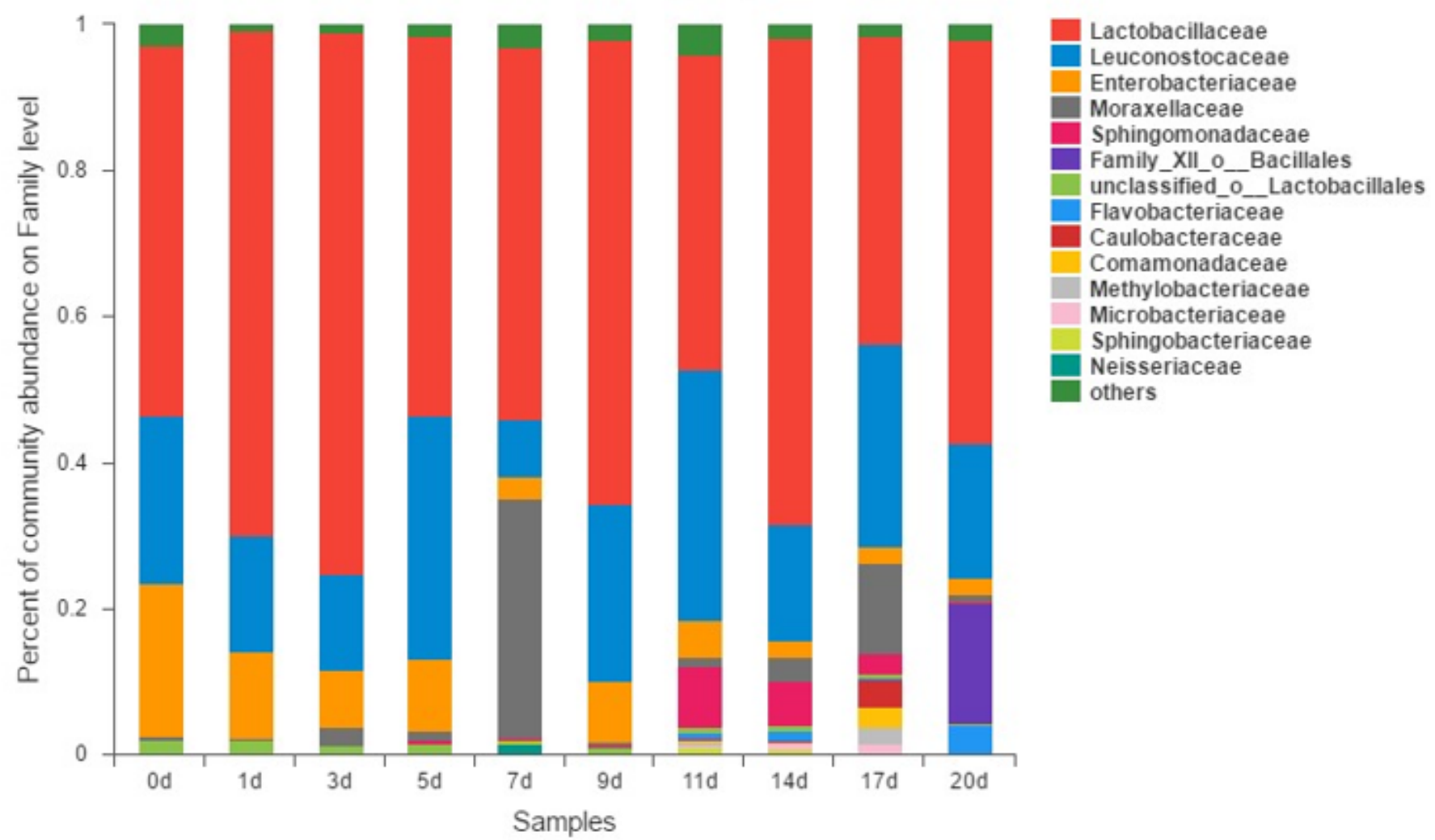

Figure 6

Relative abundance plots of bacterial community composition during the fermentation on family level. 


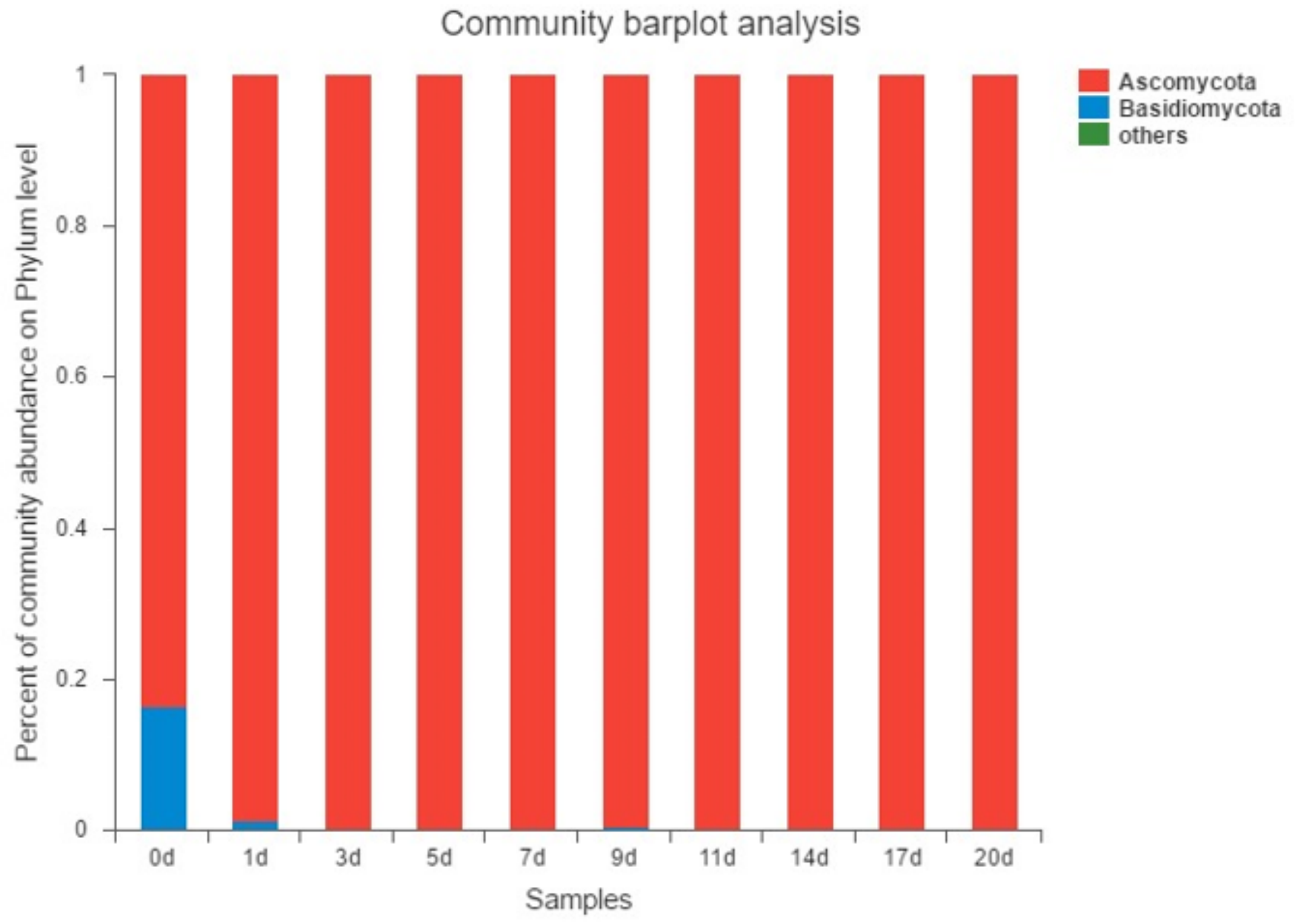

Figure 7

Relative abundance plots of fungal community composition during the fermentation on phylum level. 
Community barplot analysis

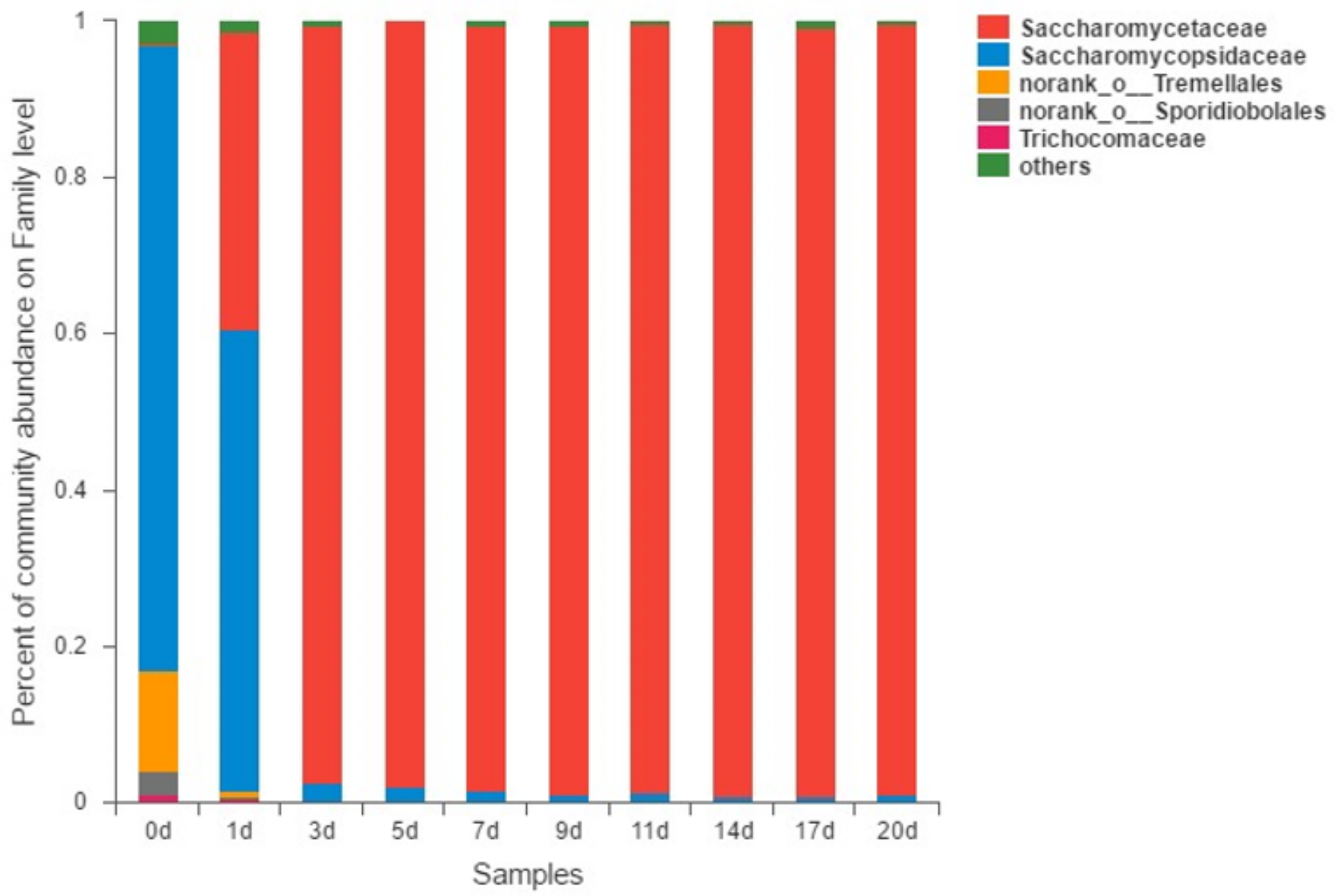

Figure 8

Relative abundance plots of fungal community composition during the fermentation at family level. 


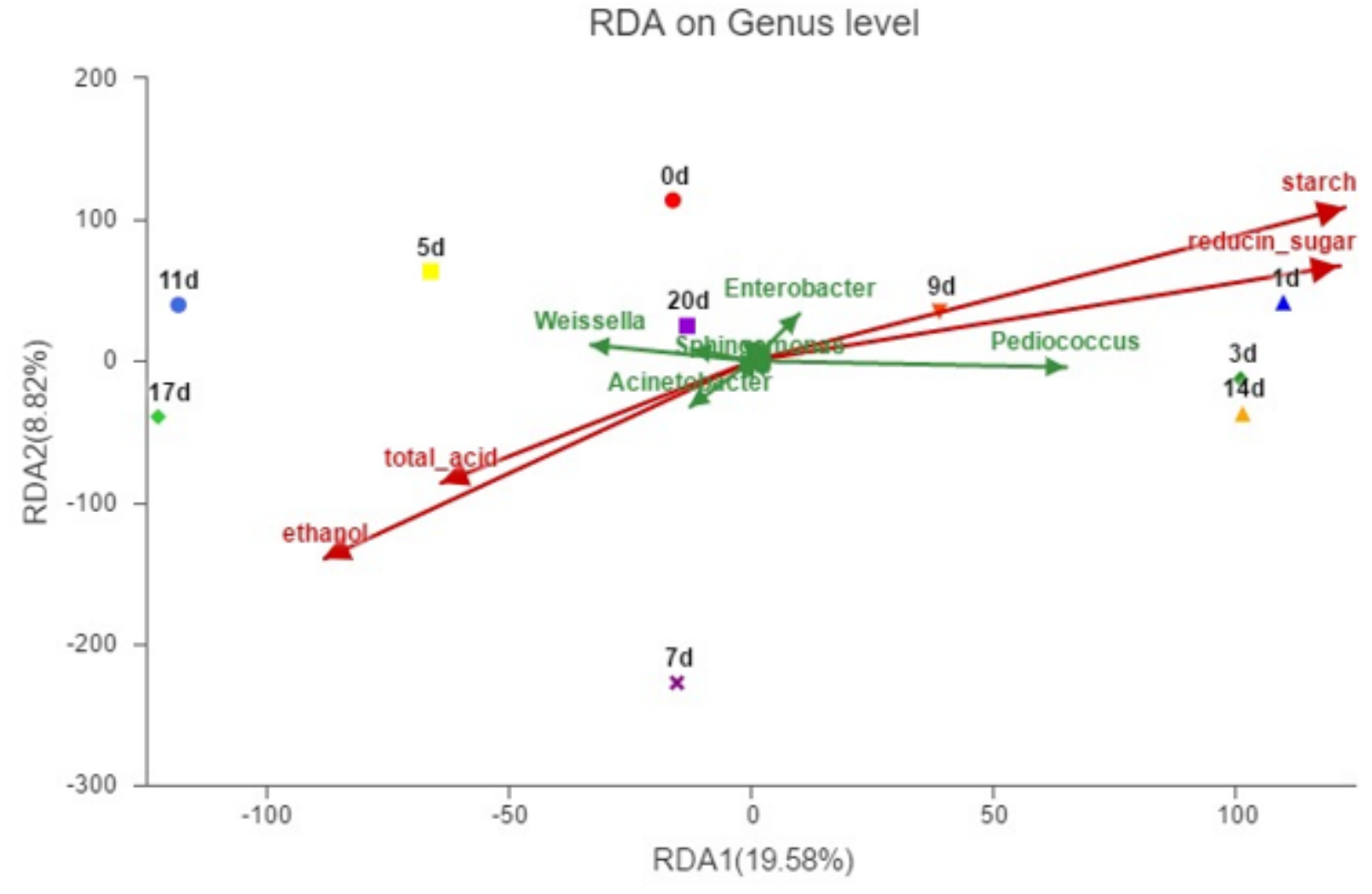

Figure 9

RDA analysis of bacterial community 


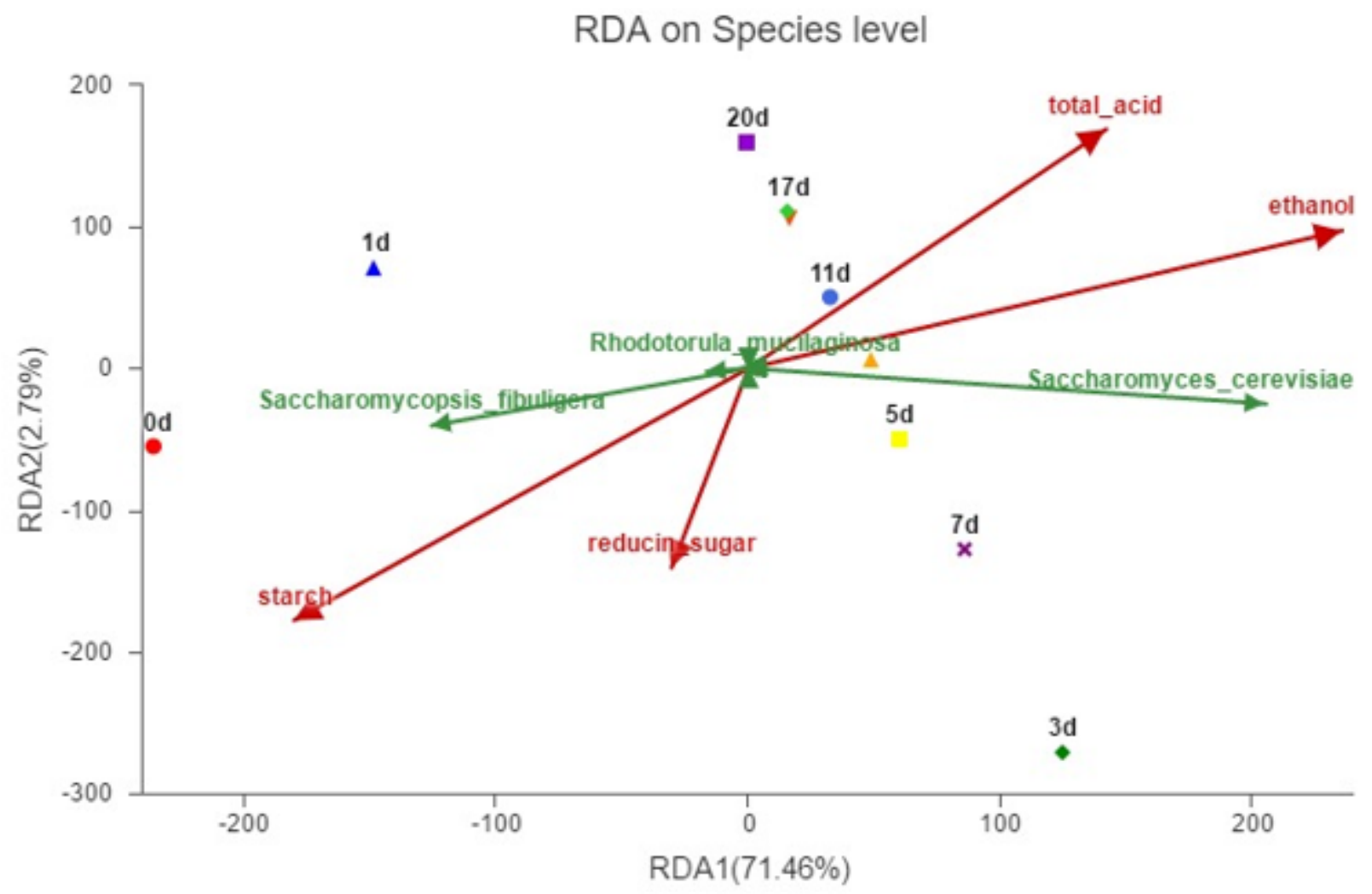

Figure 10

RDA analysis of fungal community 